\title{
Bifurcation of a Delayed SEIS Epidemic Model with a Changing Delitescence and Nonlinear Incidence Rate
}

\author{
Juan Liu \\ Department of Mathematics and Physics, Bengbu University, Bengbu 233030, China \\ Correspondence should be addressed to Juan Liu; liujuan7216@163.com
}

Received 16 February 2017; Accepted 28 March 2017; Published 9 May 2017

Academic Editor: Lu-Xing Yang

Copyright (C) 2017 Juan Liu. This is an open access article distributed under the Creative Commons Attribution License, which permits unrestricted use, distribution, and reproduction in any medium, provided the original work is properly cited.

This paper is concerned with a delayed SEIS (Susceptible-Exposed-Infectious-Susceptible) epidemic model with a changing delitescence and nonlinear incidence rate. First of all, local stability of the endemic equilibrium and the existence of a Hopf bifurcation are studied by choosing the time delay as the bifurcation parameter. Directly afterwards, properties of the Hopf bifurcation are determined based on the normal form theory and the center manifold theorem. At last, numerical simulations are carried out to illustrate the obtained theoretical results.

\section{Introduction}

The outbreak of infectious diseases had not only caused the loss of billions of lives but also badly damaged the social economy in a short time, which brought much pain to human society [1]. Thus, it has been an increasingly urgent issue to understand how to prevent or slow down the transmission of infectious diseases. To this end, many mathematical models have been proposed for describing the spread process of infectious diseases [2-10]. However, all the epidemic models above do not consider the change of delitescence of the infectious diseases. Considering that the diversity of the delitescence period in each infected individual who is infected with disease virus is mainly due to the variation of the virus and the distinct constitution of different people for some disease, such as H1N1 disease, Wang proposed the following SEIS epidemic model with a changing delitescence and a nonlinear incidence rate [11]:

$$
\begin{aligned}
& \frac{d S(t)}{d t}=A-d S(t)-\frac{\beta S(t) I(t)}{1+\alpha I(t)}+\gamma I(t), \\
& \frac{d E(t)}{d t}=\mu \frac{\beta S(t) I(t)}{1+\alpha I(t)}-(d+\varepsilon) E(t),
\end{aligned}
$$

$$
\begin{aligned}
\frac{d I(t)}{d t}= & (1-\mu) \frac{\beta S(t) I(t)}{1+\alpha I(t)}+\varepsilon E(t) \\
& -(d+\gamma+\delta) I(t),
\end{aligned}
$$

where $S(t), E(t)$, and $I(t)$ denote the numbers of the susceptible, exposed, and infectious populations at time $t$, respectively. $A$ is the recruitment rate of the susceptible population; $d$ is the natural death rate of the population; $\delta$ is the death rate due to the disease of the infected population; $\varepsilon$ is the rate at which the exposed population becomes infectious; $\gamma$ is the rate at which the infected population returns to the susceptible population because of the treatment; $\mu$ is the rate at which the infected population becomes the exposed one; and $1-\mu$ is the rate at which the infected population becomes infectious directly. $\beta S I /(1+\alpha I)$ is the nonlinear incidence rate, where $\beta$ measures the infection force of the disease and $\alpha$ measures the inhibition effect from the behavioral change of the susceptible population. Wang investigated global stability of system (1).

In fact, many infectious diseases have different kinds of delays during their spreading process in the population, such as latent period delay $[9,12-16]$, immunity period delay $[17,18]$, and infection period delay [19]. The time delay may induce Hopf bifurcation and periodic solutions. The 
occurrence of a Hopf bifurcation means that the state of the epidemic disease prevalence changes from an equilibrium to a limit cycle. Therefore, the time delay can influence the dynamics of infectious diseases. So it is necessary and useful to investigate system (1) with time delay. Based on this fact and taking the period used to cure the infectious population, we consider the following delayed epidemic system:

$$
\begin{aligned}
\frac{d S(t)}{d t}= & A-d S(t)-\frac{\beta S(t) I(t)}{1+\alpha I(t)}+\gamma I(t-\tau), \\
\frac{d E(t)}{d t}= & \mu \frac{\beta S(t) I(t)}{1+\alpha I(t)}-(d+\varepsilon) E(t), \\
\frac{d I(t)}{d t}= & (1-\mu) \frac{\beta S(t) I(t)}{1+\alpha I(t)}+\varepsilon E(t)-(d+\delta) I(t) \\
& -\gamma I(t-\tau),
\end{aligned}
$$

where $\tau$ is the time delay due to the period that is used to cure the infectious population. That is, we assume that all the infectious populations will survive after time $\tau$. The initial conditions for system (2) are

$$
\begin{aligned}
\left(\phi_{1}(\theta), \phi_{2}(\theta), \phi_{3}(\theta)\right) \in C & =C\left([-\tau, 0], R_{+}^{3}\right), \\
\phi_{1}(\theta) & >0, \phi_{2}(\theta)>0, \phi_{3}(\theta)>0,
\end{aligned}
$$

where $R_{+}^{3}=(S, E, I) \in R_{+}^{3}$.

The outline of this paper is as follows. In the next section, stability of the endemic equilibrium is analyzed and the critical value of the time delay at which a Hopf bifurcation occurs is obtained. In Section 3, direction and stability of the Hopf bifurcation are investigated. In Section 4, the obtained theoretical results are verified by some numerical simulations. Finally, this work is summarized in Section 5.

\section{Stability of the Endemic Equilibrium and Existence of Hopf Bifurcation}

By a direct computation, we know that if (I) $b_{2}=0$ and $b_{0} / b_{1}<$ 0 , (II) $b_{1}^{2}-4 b_{0} b_{2}>0$ and $b_{0} / b_{2}<0$, (III) $b_{1}^{2}-4 b_{0} b_{2}>0, b_{0}=0$ and $b_{1} / b_{2}<0$, or (IV) $b_{1}^{2}-4 b_{0} b_{2}=0$ and $b_{1} / b_{2}<0$, then system (2) has a unique endemic equilibrium $P_{*}\left(S_{*}, E_{*}, I_{*}\right)$, where

$$
\begin{aligned}
& S_{*}=\frac{(d+\varepsilon)(d+\gamma+\delta)\left(1+\alpha I_{*}\right)}{\beta(1-\mu)(d+\varepsilon)+\mu \beta \varepsilon}, \\
& E_{*}=\frac{\left(\mu \beta S_{*} I_{*}\right)}{\left((d+\varepsilon)\left(1+\alpha I_{*}\right)\right)},
\end{aligned}
$$

and $I_{*}$ is the unique positive root of the following equation:

$$
b_{2} I^{2}+b_{1} I+b_{0}=0
$$

where

$$
\begin{aligned}
b_{0}= & d(d+\varepsilon)(d+\gamma+\varepsilon)-A \beta((1-\mu)(d+\varepsilon)+\mu \varepsilon), \\
b_{1}= & (d+\varepsilon)(2 d \alpha+\beta)(d+\gamma+\varepsilon) \\
& -\beta((1-\mu)(d+\varepsilon)+\mu \varepsilon)(A \alpha+\gamma), \\
b_{2}= & \alpha(d+\varepsilon)(d \alpha+\beta)(d+\gamma+\varepsilon) .
\end{aligned}
$$

Let $u_{1}(t)=S(t)-S_{*}, u_{2}(t)=E(t)-E_{*}, u_{3}(t)=I(t)-I_{*}$. We can rewrite system (2) as the following form:

$$
\begin{aligned}
\dot{u}_{1}(t)= & a_{11} u_{1}(t)+a_{13} u_{3}(t)+b_{13} u_{3}(t-\tau) \\
& +\sum_{i+j \geq 2} \frac{1}{i ! j !} f_{i j}^{(1)} u_{1}^{i}(t) u_{3}^{j}(t), \\
\dot{u}_{2}(t)= & a_{21} u_{1}(t)+a_{22} u_{2}(t)+a_{23} u_{3}(t) \\
& +\sum_{i+j \geq 2} \frac{1}{i ! j !} f_{i j}^{(2)} u_{1}^{i}(t) u_{3}^{j}(t), \\
\dot{u}_{3}(t)= & a_{31} u_{1}(t)+a_{32} u_{2}(t)+a_{33} u_{3}(t)+b_{33} u_{3}(t-\tau) \\
& +\sum_{i+j \geq 2} \frac{1}{i ! j !} f_{i j}^{(3)} u_{1}^{i}(t) u_{3}^{j}(t),
\end{aligned}
$$

where

$$
\begin{aligned}
& a_{11}=-\left(d+\frac{\beta I_{*}}{1+\alpha I_{*}}\right), \\
& a_{13}=-\frac{\beta S_{*}}{1+\alpha I_{*}}, \\
& b_{13}=\gamma \\
& a_{21}=\frac{\mu \beta I_{*}}{1+\alpha I_{*}}, \\
& a_{22}=-(d+\varepsilon), \\
& a_{23}=\frac{\mu \beta S_{*}}{1+\alpha I_{*}}, \\
& a_{31}=\frac{(1-\mu) \beta I_{*}}{1+\alpha I_{*}}, \\
& a_{32}=\varepsilon, \\
& a_{33}=\frac{(1-\mu) \beta I_{*}}{1+\alpha I_{*}}-(d+\delta),
\end{aligned}
$$




$$
\begin{aligned}
b_{33}= & -\gamma, \\
f_{i j}^{(k)}= & \frac{\partial^{i+j} f^{(k)}\left(S_{*}, E_{*}, I_{*}\right)}{\partial u_{1}^{i}(t) \partial u_{3}^{j}(t)}, \\
f^{(1)}= & A-d u_{1}(t)-\frac{\beta u_{1}(t) u_{3}(t)}{1+\alpha u_{3}(t)}+\gamma u_{3}(t-\tau), \\
f^{(2)}= & \mu \frac{\beta u_{1}(t) u_{3}(t)}{1+\alpha u_{3}(t)}-(d+\varepsilon) u_{2}(t), \\
f^{(3)}= & (1-\mu) \frac{\beta u_{1}(t) u_{3}(t)}{1+\alpha u_{3}(t)}+\varepsilon u_{2}(t)-(d+\delta) u_{3}(t) \\
& -\gamma u_{3}(t-\tau) .
\end{aligned}
$$

Then we obtain the linearized system of system (2)

$$
\begin{aligned}
\dot{u}_{1}(t)= & a_{11} u_{1}(t)+a_{13} u_{3}(t)+b_{13} u_{3}(t-\tau), \\
\dot{u}_{2}(t)= & a_{21} u_{1}(t)+a_{22} u_{2}(t)+a_{23} u_{3}(t), \\
\dot{u}_{3}(t)= & a_{31} u_{1}(t)+a_{32} u_{2}(t)+a_{33} u_{3}(t) \\
& +b_{33} u_{3}(t-\tau) .
\end{aligned}
$$

The characteristic equation is

$$
\begin{aligned}
\lambda^{3} & +A_{2} \lambda^{2}+A_{1} \lambda+A_{0}+\left(B_{2} \lambda^{2}+B_{1} \lambda+B_{0}\right) e^{-\lambda \tau} \\
& =0
\end{aligned}
$$

where

$$
\begin{aligned}
& A_{0}=a_{13}\left(a_{22} a_{31}-a_{21} a_{32}\right)+a_{11}\left(a_{23} a_{32}-a_{22} a_{33}\right), \\
& A_{1}=a_{11} a_{22}+a_{22} a_{33}+a_{11} a_{33} \\
& A_{2}=-\left(a_{11}+a_{22}+a_{33}\right) \\
& B_{0}=b_{13}\left(a_{22} a_{31}-a_{21} a_{32}\right)-a_{11} a_{22} b_{33} \\
& B_{1}=b_{33}\left(a_{11}+a_{22}\right)-a_{13} b_{13} \\
& B_{2}=-b_{33} .
\end{aligned}
$$

When $\tau=0$, (10) reduces to

$$
\lambda^{3}+\left(A_{2}+B_{2}\right) \lambda^{2}+\left(A_{1}+B_{1}\right) \lambda+A_{0}+B_{0}=0 .
$$

Routh-Hurwitz criterion implies that $P_{*}$ is locally asymptotically stable without delay if condition $\left(H_{1}\right)$ holds.

$\left(H_{1}\right) A_{2}+B_{2}>0,\left(A_{2}+B_{2}\right)\left(A_{1}+B_{1}\right)>A_{0}+B_{0}>0$.

For $\tau>0$, substituting $\lambda=i \omega(\omega>0)$ into (10), we obtain

$$
\begin{aligned}
& B_{1} \omega \sin \tau \omega+\left(B_{0}-B_{2} \omega^{2}\right) \cos \tau \omega=A_{2} \omega^{2}-A_{0}, \\
& B_{1} \omega \cos \tau \omega-\left(B_{0}-B_{2} \omega^{2}\right) \sin \tau \omega=\omega^{3}-A_{1} \omega .
\end{aligned}
$$

Then

$$
\omega^{6}+a_{2} \omega^{4}+a_{1} \omega^{2}+a_{0}=0
$$

where

$$
\begin{aligned}
& a_{0}=A_{0}^{2}-B_{0}^{2}, \\
& a_{1}=A_{1}^{2}-2 A_{0} A_{2}-B_{1}^{2}+2 B_{0} B_{2}, \\
& A_{2}=A_{2}^{2}-2 A_{1}-B_{2}^{2} .
\end{aligned}
$$

Let $\omega^{2}=v$; then

$$
v^{3}+a_{2} v^{2}+a_{1} v+a_{0}=0
$$

where $f(v)=v^{3}+a_{2} v^{2}+a_{1} v+a_{0}$. According to the analysis about the distribution of roots of (16) in Song et al. [20], we have the following result.

Lemma 1. For the polynomial equation (16),

(1) if $a_{0}<0$, then (16) has at least one positive root;

(2) if $a_{0} \geq 0$ and $\triangle=a_{2}^{2}-3 a_{1} \leq 0$, then (16) has no positive roots;

(3) if $a_{0} \geq 0$ and $\Delta=a_{2}^{2}-3 a_{1}>0$, then (16) has positive roots if and only if $v_{1}^{*}=\left(-a_{2}+\sqrt{\triangle}\right) / 3>0$ and $f\left(v_{1}^{*}\right) \leq$ 0 .

Next, we assume that the coefficients in (16) satisfy the following condition.

$\left(H_{2}\right)$ (i) $a_{0}<0$ or (ii) $a_{0} \geq 0, \Delta=a_{2}^{2}-3 a_{1}>0, v_{1}^{*}=$ $\left(-a_{2}+\sqrt{\triangle}\right) / 3>0$, and $f\left(v_{1}^{*}\right) \leq 0$.

Thus, (14) has at least one positive root such that (10) has a pair of purely imaginary roots $\pm i \omega_{0}$. The corresponding critical value $\tau_{0}$ can be obtained from (13)

$$
\begin{aligned}
\tau_{0} & =\frac{1}{\omega_{0}} \\
& \cdot \arccos \frac{\left(B_{1}-A_{2} B_{2}\right) \omega_{0}^{4}+\left(A_{2} B_{0}+A_{0} B_{2}-A_{1} B_{1}\right) \omega_{0}^{2}-A_{0} B_{0}}{B_{1}^{2} \omega_{0}^{2}+\left(B_{0}-B_{2} \omega_{0}^{2}\right)^{2}} .
\end{aligned}
$$

Taking derivative with respect to $\tau$ on both sides of (10), we obtain

$$
\begin{aligned}
{\left[\frac{d \lambda}{d \tau}\right]^{-1}=} & -\frac{3 \lambda^{2}+2 A_{2} \lambda+A_{1}}{\lambda\left(\lambda^{3}+A_{2} \lambda^{2}+A_{1} \lambda+A_{0}\right)} \\
& +\frac{2 B_{2} \lambda+B_{1}}{\lambda\left(B_{2} \lambda^{2}+B_{1} \lambda+B_{0}\right)}-\frac{\tau}{\lambda} .
\end{aligned}
$$

Further, we have

$$
\operatorname{Re}\left[\frac{d \lambda}{d \tau}\right]_{\tau=\tau_{0}}^{-1}=\frac{f^{\prime}\left(\omega_{0}^{2}\right)}{B_{1}^{2} \omega_{0}^{2}+\left(B_{0}-B_{2} \omega_{0}^{2}\right)^{2}}
$$

Thus, if the condition $\left(H_{3}\right): f^{\prime}\left(\omega_{0}^{2}\right) \neq 0$ holds, then $\operatorname{Re}[d \lambda / d \tau]_{\tau=\tau_{0}}^{-1} \neq 0$. Then, based on the Hopf bifurcation theorem in [21], we have the following.

Theorem 2. For system (2), if the conditions $\left(H_{1}\right)-\left(H_{3}\right)$ hold, then the endemic equilibrium $E_{*}\left(S_{*}, E_{*}, I_{*}\right)$ of system (2) is asymptotically stable for $\tau \in\left[0, \tau_{0}\right)$ and system (2) undergoes a Hopf bifurcation at the endemic equilibrium $E_{*}\left(S_{*}, E_{*}, I_{*}\right)$ when $\tau=\tau_{0}$, where $\tau_{0}$ is defined in (17). 


\section{Direction and Stability of the Hopf Bifurcation}

Let $\tau=\tau_{0}+\mu, \mu \in R$; then $\mu=0$ is the Hopf bifurcation value of system (2). Rescaling the time delay $t \rightarrow(t / \tau)$, then system (2) can be transformed into an FDE in $C=C\left([-1,0], R^{3}\right)$ as follows:

$$
\dot{u}(t)=L_{\mu} u_{t}+F\left(\mu, u_{t}\right)
$$

where

$$
\begin{aligned}
L_{\mu} \phi= & \left(\tau_{0}+\mu\right)\left(\begin{array}{ccc}
a_{11} & 0 & a_{13} \\
a_{21} & a_{22} & a_{23} \\
a_{31} & a_{32} & a_{33}
\end{array}\right) \phi(0) \\
& +\left(\tau_{0}+\mu\right)\left(\begin{array}{ccc}
0 & 0 & b_{13} \\
0 & 0 & 0 \\
0 & 0 & b_{33}
\end{array}\right) \phi(-1), \\
F(\mu, \phi)= & \left(\tau_{0}+\mu\right)\left(F_{1}, F_{2}, F_{3}\right)^{T},
\end{aligned}
$$

where $F_{1}, F_{2}$, and $F_{3}$ are defined by Appendix A.

By the Riesz representation theorem, there exists a $3 \times 3$ matrix function $\eta(\theta, \mu), \theta \in[-1,0]$, whose components are of bounded variation, such that

$$
L_{\mu} \phi=\int_{-1}^{0} d \eta(\theta, \mu) \phi(\theta), \quad \phi \in C\left([-1,0], R^{3}\right) .
$$

In fact, we choose

$$
\begin{aligned}
\eta(\theta, \mu)= & \left(\tau_{0}+\mu\right)\left(\begin{array}{ccc}
a_{11} & 0 & a_{13} \\
a_{21} & a_{22} & a_{23} \\
a_{31} & a_{32} & a_{33}
\end{array}\right) \phi(0) \\
& +\left(\tau_{0}+\mu\right)\left(\begin{array}{ccc}
0 & 0 & b_{13} \\
0 & 0 & 0 \\
0 & 0 & b_{33}
\end{array}\right) \phi(-1) .
\end{aligned}
$$

For $\phi \in C\left([-1,0], R^{3}\right)$, we define

$$
\begin{aligned}
& A(\mu) \phi= \begin{cases}\frac{d \phi(\theta)}{d \theta}, & -1 \leq \theta<0, \\
\int_{-1}^{0} d \eta(\theta, \mu) \phi(\theta), & \theta=0,\end{cases} \\
& R(\mu) \phi= \begin{cases}0, & -1 \leq \theta<0, \\
F(\mu, \phi), & \theta=0 .\end{cases}
\end{aligned}
$$

Then system (20) is equivalent to

$$
\dot{u}(t)=A(\mu) u_{t}+R(\mu) u_{t} .
$$

For $\varphi \in C^{1}([0,1]),\left(R^{3}\right)^{*}$, the adjoint operator $A^{*}$ of $A$ is defined as

$$
A^{*}(\varphi)= \begin{cases}-\frac{d \varphi(s)}{d s}, & 0<s \leq 1, \\ \int_{-1}^{0} d \eta^{T}(s, 0) \varphi(-s), & s=0,\end{cases}
$$

and a bilinear inner product is defined by

$$
\begin{aligned}
\langle\varphi(s), \phi(\theta)\rangle= & \bar{\varphi}(0) \phi(0) \\
& -\int_{\theta=-1}^{0} \int_{\xi=0}^{\theta} \bar{\varphi}(\xi-\theta) d \eta(\theta) \phi(\xi) d \xi
\end{aligned}
$$

where $\eta(\theta)=\eta(\theta, 0)$.

Let $q(\theta)=\left(1, q_{2}, q_{3}\right)^{T} e^{i \omega_{0} \tau_{0} \theta}$ be the eigenvector of $A(0)$ belonging to $+i \omega_{0} \tau_{0}$ and $q^{*}(s)=D\left(1, q_{2}^{*}, q_{3}^{*}\right) e^{i \omega_{0} \tau_{0} s}$ be the eigenvector of $A^{*}(0)$ belonging to $-i \omega_{0} \tau_{0}$. By a direct computation, we can get

$$
\begin{aligned}
& q_{2}=\frac{a_{21}+a_{23} q_{3}}{i \omega_{0}-a_{22}}, \\
& q_{3}=\frac{i \omega_{0}-a_{11}}{a_{13}+b_{13} e^{-i \tau_{0} \omega_{0}}}, \\
& q_{2}^{*}=-\frac{a_{32} q_{3}}{i \omega_{0}+a_{22}}, \\
& q_{3}^{*}=\frac{\left(a_{13}+b_{13} e^{i \tau_{0} \omega_{0}}\right)\left(i \omega_{0}+a_{22}\right)}{a_{23} a_{32}-\left(i \omega_{0}+a_{22}\right)\left(i \omega_{0}+a_{33}+b_{33} e^{i \tau_{0} \omega_{0}}\right)} .
\end{aligned}
$$

From (27), we can get

$$
\begin{aligned}
& \left\langle q^{*}(s), q(\theta)\right\rangle \\
& \quad=\bar{D}\left[1+q_{2} \bar{q}_{2}^{*}+q_{3} \bar{q}_{3}^{*}+\tau_{0} e^{-i \tau_{0} \omega_{0}} q_{3}\left(b_{13}+b_{33} \bar{q}_{3}^{*}\right)\right] .
\end{aligned}
$$

Then we choose

$$
\bar{D}=\left[1+q_{2} \bar{q}_{2}^{*}+q_{3} \bar{q}_{3}^{*}+\tau_{0} e^{-i \tau_{0} \omega_{0}} q_{3}\left(b_{13}+b_{33} \bar{q}_{3}^{*}\right)\right]^{-1} .
$$

such that $\left\langle q^{*}, q\right\rangle=1$.

Next, we can obtain the coefficients $g_{20}, g_{11}, g_{02}$, and $g_{21}$ by using the method introduced in [21] and a computation process similar to that in [22-24]. The expressions of $g_{20}, g_{11}$, $g_{02}$, and $g_{21}$ are defined by Appendix B.

Then, we can get the following coefficients which determine the properties of the Hopf bifurcation:

$$
\begin{aligned}
C_{1}(0) & =\frac{i}{2 \tau_{0} \omega_{0}}\left(g_{11} g_{20}-2\left|g_{11}\right|^{2}-\frac{\left|g_{02}\right|^{2}}{3}\right)+\frac{g_{21}}{2}, \\
\mu_{2} & =-\frac{\operatorname{Re}\left\{C_{1}(0)\right\}}{\operatorname{Re}\left\{\lambda^{\prime}\left(\tau_{0}\right)\right\}} \\
\beta_{2} & =2 \operatorname{Re}\left\{C_{1}(0)\right\} \\
T_{2} & =-\frac{\operatorname{Im}\left\{C_{1}(0)\right\}+\mu_{2} \operatorname{Im}\left\{\lambda^{\prime}\left(\tau_{0}\right)\right\}}{\tau_{0} \omega_{0}} .
\end{aligned}
$$

In conclusion, we have the following results.

Theorem 3. For system (2), if $\mu_{2}>0\left(\mu_{2}<0\right)$, then the Hopf bifurcation is supercritical (subcritical). If $\beta_{2}<0\left(\beta_{2}>0\right)$, then the bifurcating periodic solutions are stable (unstable). If $T_{2}>0\left(T_{2}<0\right)$, then the bifurcating periodic solutions increase (decrease). 

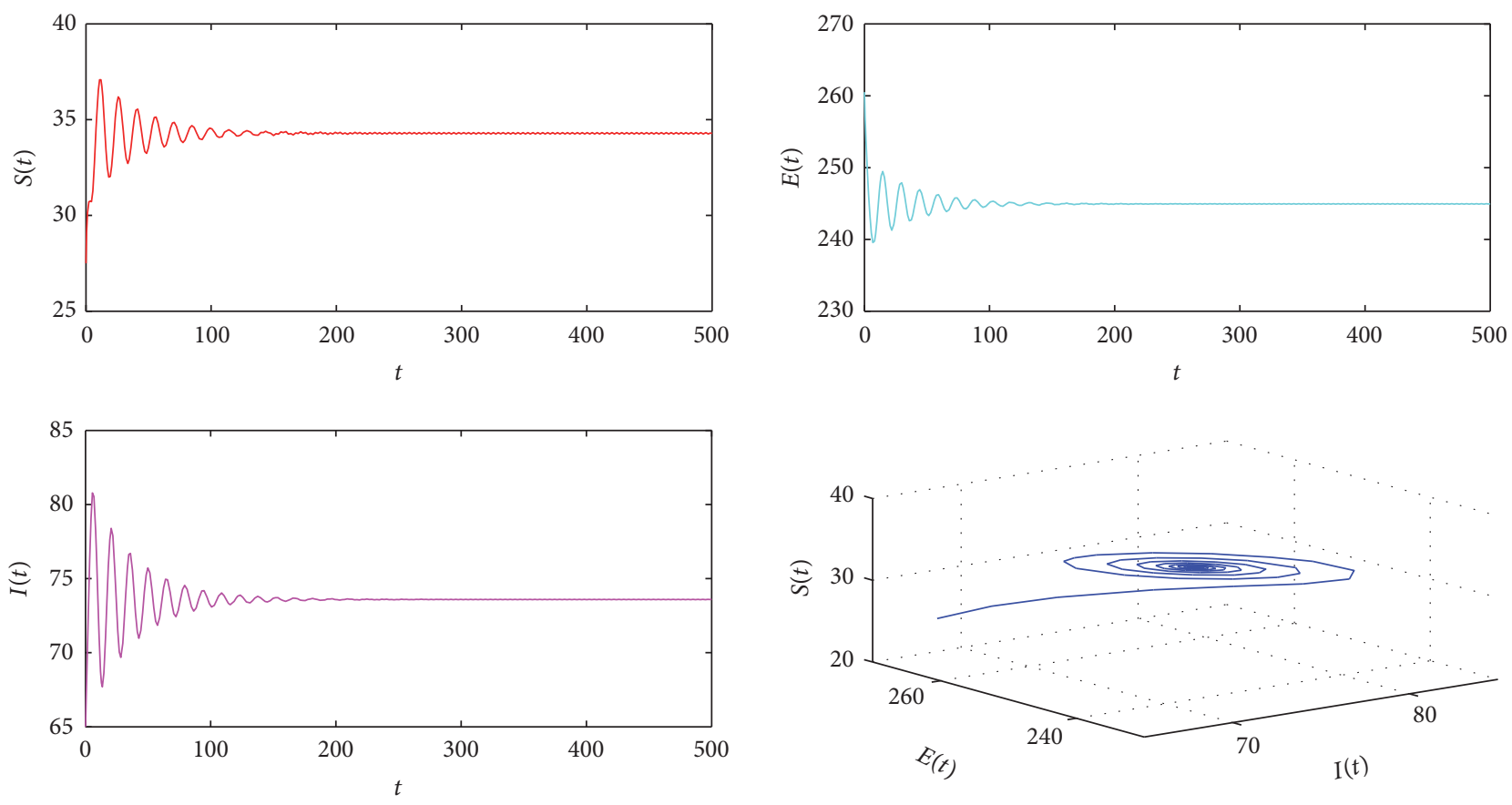

FIgURE 1: $P_{*}$ is locally asymptotically stable for $\tau=4.475<\tau_{0}=5.1686$ with initial values " $27.5,260.5,65$."

\section{Numerical Simulations}

In order to verify the efficiency of the obtained results in the paper, we carry out some numerical simulations in this section. By extracting some values from [11] and considering the conditions for the existence of the Hopf bifurcation, we consider the special case of system (2) with the parameters $A=5, d=0.01, \beta=0.5, \alpha=0.4, \gamma=0.5, \mu=0.65, \varepsilon=0.1$, and $\delta=0.02$, Then, system (2) becomes the following form:

$$
\begin{aligned}
& \frac{d S(t)}{d t}=5-0.01 S(t)-\frac{0.5 S(t) I(t)}{1+0.4 I(t)}+0.5 I(t-\tau), \\
& \frac{d E(t)}{d t}=\frac{0.325 S(t) I(t)}{1+0.4 I(t)}-0.11 E(t), \\
& \frac{d I(t)}{d t} \\
& \quad \frac{0.175 S(t) I(t)}{1+0.4 I(t)}+0.1 E(t)-0.03 I(t) \\
& \quad-0.5 I(t-\tau), \\
& 0.0014 I^{2}-0.0999 I-0.2584=0,
\end{aligned}
$$

from which we can obtain the unique positive root $I_{*}=$ 73.8562 and then we get the unique endemic equilibrium $P_{*}(34.3750,245.5930,73.8562)$. Then, we can obtain $\omega_{0}=$ $0.3950, \tau_{0}=5.1686$, and $\lambda^{\prime}\left(\tau_{0}\right)=0.0012-0.0759 i$. Thus, based on Theorem 2, we know that the endemic equilibrium $P_{*}(34.3750,245.5930,73.8562)$ is locally asymptotically stable when $\tau<\tau_{0}=5.1686$, which can be illustrated by Figures 1 and 2 . In this case, the disease can be controlled easily. Once the value of the delay passes through the critical value $\tau_{0}=5.1686$, then the endemic equilibrium $P_{*}(34.3750,245.5930,73.8562)$ loses its stability and a Hopf bifurcation occurs, and a family of periodic solutions bifurcate from the endemic equilibrium $P_{*}(34.3750,245.5930,73.8562)$. This property can be shown as in Figures 3 and 4 . In this case, the disease will be out of control.

In addition, according to (31), we get $C_{1}(0)=-1.0027-$ $0.9244 i, \mu_{2}=835.5833>0, \beta_{2}=-2.0054<0$, and $T_{2}=31.5171>0$. Therefore, we can conclude that the Hopf bifurcation is supercritical and the bifurcating periodic solutions are stable and increase. Since the bifurcating periodic solutions are stable, it can be concluded that the populations in system (32) can coexist from the view of ecology. Based on this fact, we can conclude that the time delay is harmful for system (32).

\section{Conclusions}

We generalize a delayed SEIS (Susceptible-Exposed-Infectious-Susceptible) epidemic model with a changing delitescence and nonlinear incidence rate in this paper by introducing the time delay due to the period that is used to cure the infectious population into the SEIS model considered in the literature [11]. Compared with the literature [11], we mainly consider the effect of the time delay on the model.

The main results are given in terms of local stability and Hopf bifurcation. Stability of the endemic equilibrium is investigated by analyzing the corresponding characteristic equation. By choosing the time delay as a bifurcation parameter, sufficient conditions have been established for local existence of Hopf bifurcation at the endemic equilibrium. Then, with the help of the normal form theory and the 

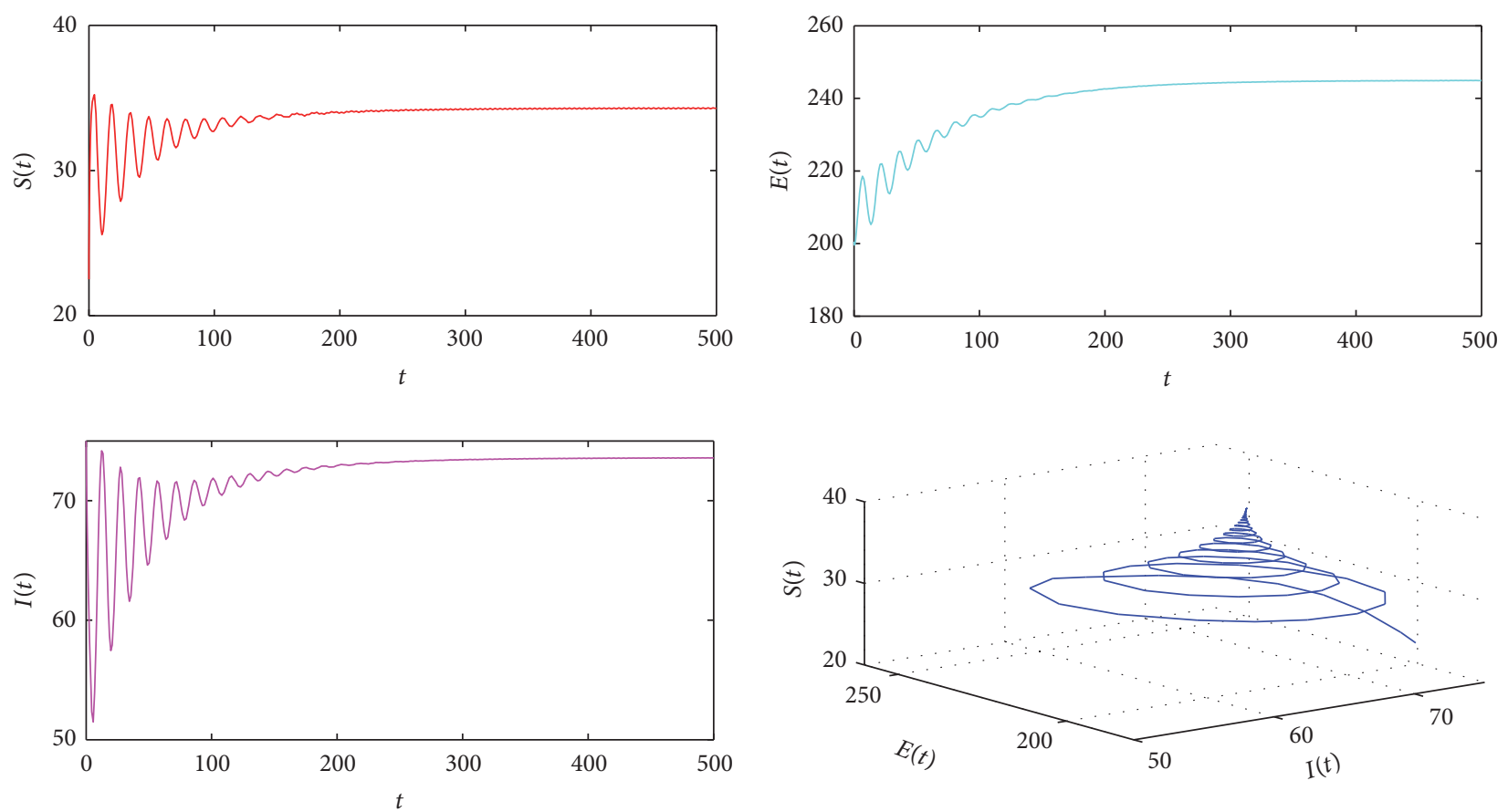

FIGURE $2: P_{*}$ is locally asymptotically stable for $\tau=4.475<\tau_{0}=5.1686$ with initial values “22, 200, 75.”
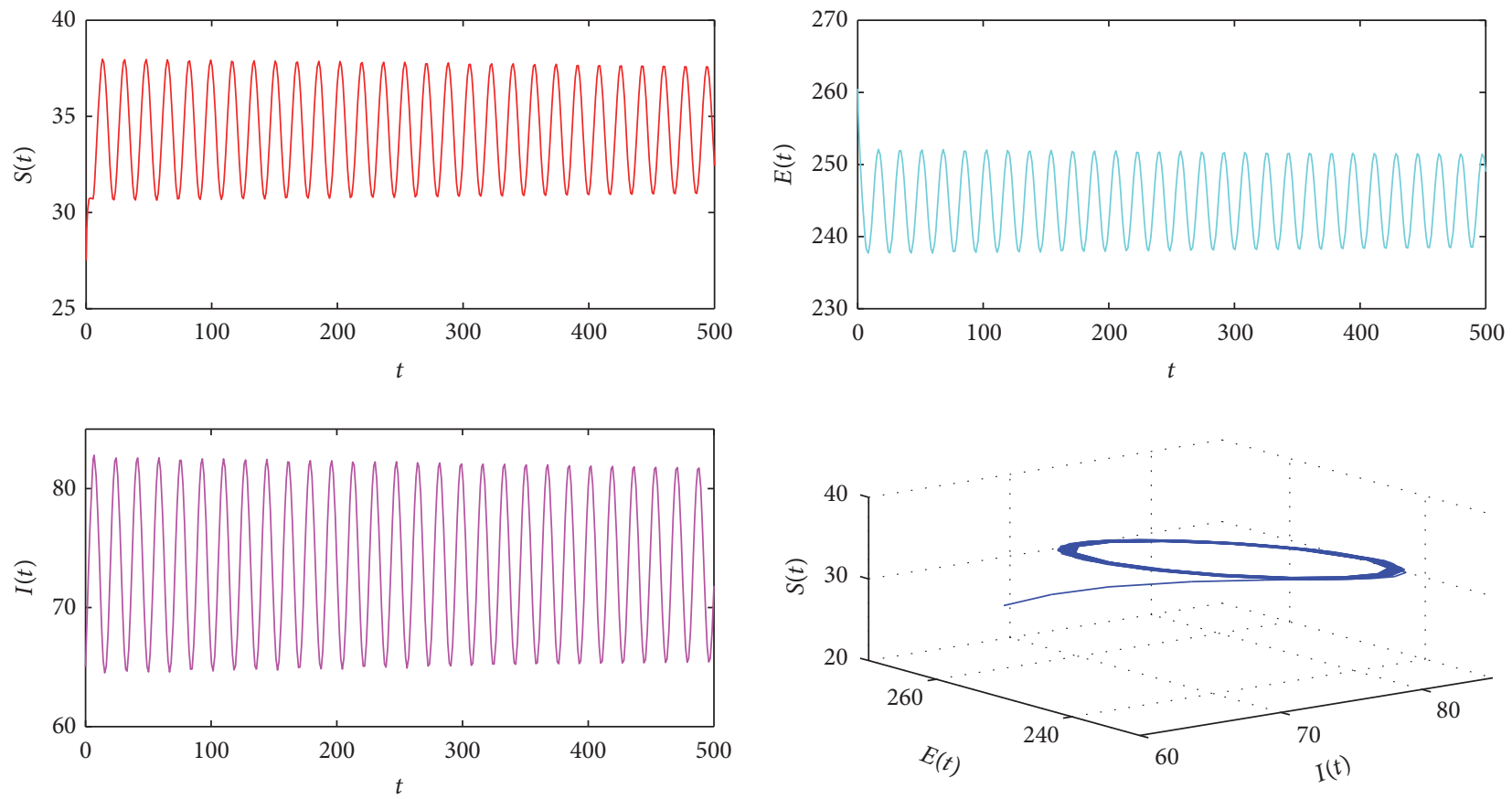

Figure 3: $P_{*}$ becomes unstable and a Hopf bifurcation occurs when $\tau=5.485>\tau_{0}=5.1686$ with initial values “27.5, 260.5, 65."

center manifold theorem due to Hassard et al. [21], direction and stability of the Hopf bifurcation are determined. Finally, through numerical simulations, it can be concluded that the period used to cure the infectious population plays an important role in the disease spreading and the disease may be controlled by shortening the period used to cure the infectious population.

\section{Appendix}

\section{A. The Expressions of $F_{1}, F_{2}$, and $F_{3}$}

$$
\begin{aligned}
F_{1}= & g_{1} \phi_{3}^{2}(0)+g_{2} \phi_{1}(0) \phi_{3}(0)+g_{3} \phi_{1}(0) \phi_{3}^{2}(0) \\
& +g_{4} \phi_{3}^{3}(0)+\cdots
\end{aligned}
$$



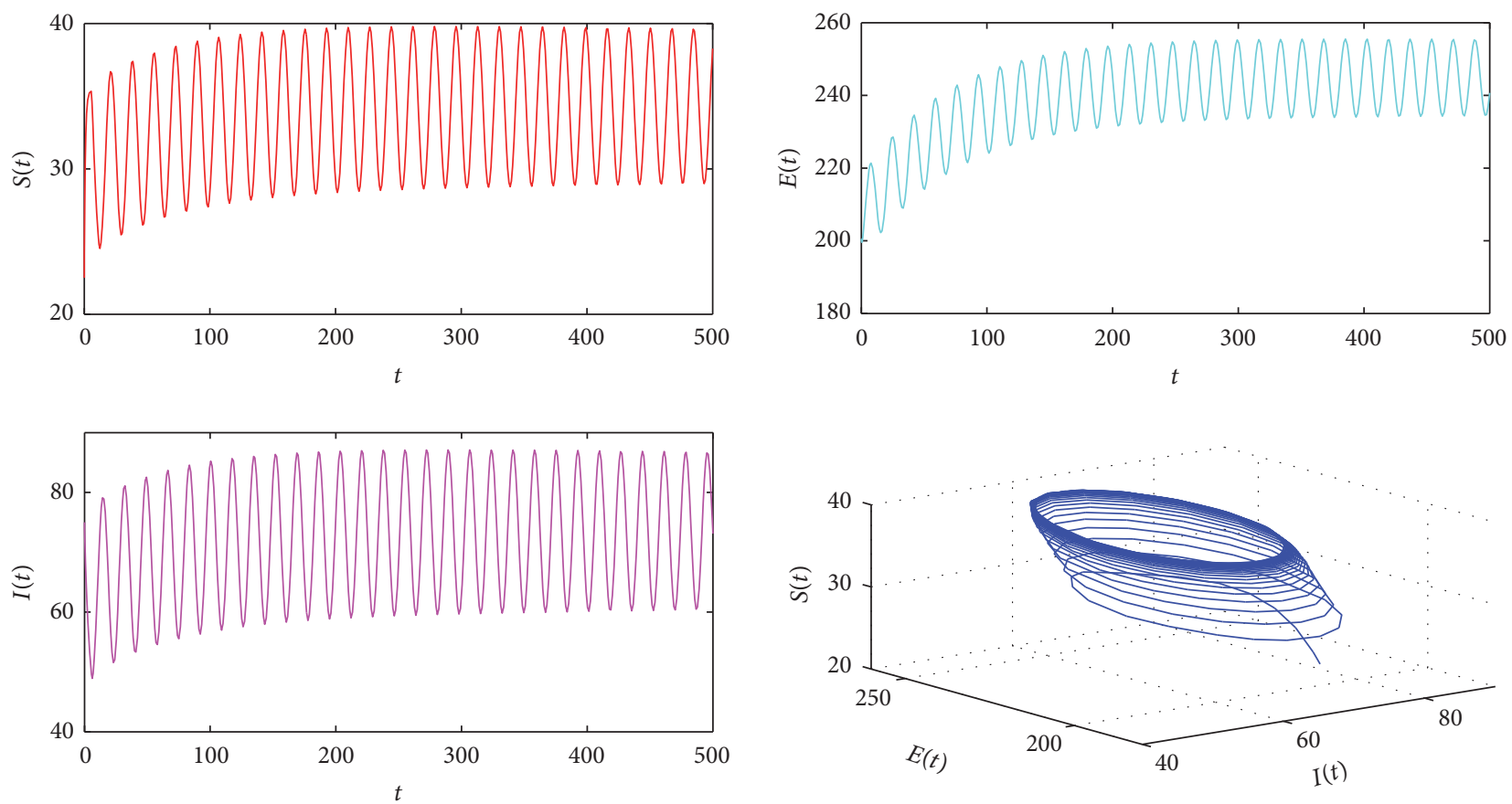

FIgURE 4: $P_{*}$ becomes unstable and a Hopf bifurcation occurs when $\tau=5.485>\tau_{0}=5.1686$ with initial values "22, 200, 75."

$$
\begin{aligned}
& F_{2}=h_{1} \phi_{3}^{2}(0)+h_{2} \phi_{1}(0) \phi_{3}(0)+h_{3} \phi_{1}(0) \phi_{3}^{2}(0) \\
& +h_{4} \phi_{3}^{3}(0)+\cdots, \\
& F_{3}=k_{1} \phi_{3}^{2}(0)+k_{2} \phi_{1}(0) \phi_{3}(0)+k_{3} \phi_{1}(0) \phi_{3}^{2}(0) \\
& +k_{4} \phi_{3}^{3}(0)+\cdots, \\
& g_{1}=\frac{\alpha \beta S_{*}}{\left(1+\alpha I_{*}\right)^{3}} \text {, } \\
& g_{2}=-\frac{\beta}{\left(1+\alpha I_{*}\right)^{2}} \text {, } \\
& g_{3}=\frac{\alpha \beta}{\left(1+\alpha I_{*}\right)^{3}} \text {, } \\
& g_{4}=-\frac{\alpha^{2} \beta S_{*}}{\left(1+\alpha I_{*}\right)^{4}} \text {, } \\
& h_{1}=-\frac{\mu \alpha \beta S_{*}}{\left(1+\alpha I_{*}\right)^{3}} \text {, } \\
& h_{2}=\frac{\mu \beta}{\left(1+\alpha I_{*}\right)^{2}} \text {, } \\
& h_{3}=-\frac{\mu \alpha \beta}{\left(1+\alpha I_{*}\right)^{3}} \text {, } \\
& h_{4}=\frac{\mu \alpha^{2} \beta S_{*}}{\left(1+\alpha I_{*}\right)^{4}} \text {, }
\end{aligned}
$$

$$
\begin{aligned}
& k_{1}=-\frac{(1-\mu) \alpha \beta S_{*}}{\left(1+\alpha I_{*}\right)^{3}}, \\
& k_{2}=\frac{(1-\mu) \beta}{\left(1+\alpha I_{*}\right)^{2}}, \\
& k_{3}=-\frac{(1-\mu) \alpha \beta}{\left(1+\alpha I_{*}\right)^{3}}, \\
& k_{4}=\frac{(1-\mu) \alpha^{2} \beta S_{*}}{\left(1+\alpha I_{*}\right)^{4}} .
\end{aligned}
$$

B. The Expressions of $g_{20}, g_{11}, g_{02}$, and $g_{21}$

$$
\begin{aligned}
g_{20} & =2 \tau_{0} \bar{D}\left(g_{1}\left(q^{(3)}(0)\right)^{2}+g_{2} q^{(1)}(0) q^{(3)}(0)\right. \\
& +\bar{q}_{2}^{*}\left(h_{1}\left(q^{(3)}(0)\right)^{2}+h_{2} q^{(1)}(0) q^{(3)}(0)\right)+\bar{q}_{3}^{*}\left(k_{1}\left(q^{(3)}(0)\right)^{2}\right. \\
& \left.\left.+k_{2} q^{(1)}(0) q^{(3)}(0)\right)\right), \\
g_{11} & =\tau_{0} \bar{D}\left(2 g_{1} q^{(3)}(0) \bar{q}^{(3)}(0)+g_{2}\left(q^{(1)}(0) \bar{q}^{(3)}(0)+\bar{q}^{(1)}(0)\right.\right. \\
& \left.\cdot q^{(3)}(0)\right)+\bar{q}_{2}^{*}\left(2 h_{1} q^{(3)}(0) \bar{q}^{(3)}(0)+h_{2}\left(q^{(1)}(0) \bar{q}^{(3)}(0)\right.\right. \\
& \left.\left.+\bar{q}^{(1)}(0) q^{(3)}(0)\right)\right)+\bar{q}_{3}^{*}\left(2 k_{1} q^{(3)}(0) \bar{q}^{(3)}(0)\right. \\
& \left.\left.+k_{2}\left(q^{(1)}(0) \bar{q}^{(3)}(0)+\bar{q}^{(1)}(0) q^{(3)}(0)\right)\right)\right) \\
g_{02} & =2 \tau_{0} \bar{D}\left(g_{1}\left(\bar{q}^{(3)}(0)\right)^{2}+g_{2} \bar{q}^{(1)}(0) \bar{q}^{(3)}(0)\right.
\end{aligned}
$$




$$
\begin{aligned}
& +\bar{q}_{2}^{*}\left(h_{1}\left(\bar{q}^{(3)}(0)\right)^{2}+h_{2} \bar{q}^{(1)}(0) \bar{q}^{(3)}(0)\right)+\bar{q}_{3}^{*}\left(k_{1}\left(\bar{q}^{(3)}(0)\right)^{2}\right. \\
& \left.\left.+k_{2} \bar{q}^{(1)}(0) \bar{q}^{(3)}(0)\right)\right) \\
& g_{21}=2 \tau_{0} \bar{D}\left(g_{1}\left(2 W_{11}^{(3)}(0) q^{(3)}(0)+W_{20}^{(3)}(0) \bar{q}^{(3)}(0)\right)\right. \\
& +g_{2}\left(W_{11}^{(1)}(0) q^{(3)}(0)+\frac{1}{2} W_{20}^{(1)}(0) \bar{q}^{(3)}(0)+W_{11}^{(3)}(0) q^{(1)}(0)\right. \\
& \left.+\frac{1}{2} W_{20}^{(3)}(0) \bar{q}^{(1)}(0)\right)+g_{3}\left(\bar{q}^{(1)}(0)\left(q^{(3)}(0)\right)^{2}+2 q^{(1)}(0)\right. \\
& \left.\cdot q^{(3)}(0) \bar{q}^{(3)}(0)\right)+3 g_{4}\left(q^{(3)}(0)\right)^{2} \bar{q}^{(3)}(0) \\
& +\bar{q}_{2}^{*}\left(h_{1}\left(2 W_{11}^{(3)}(0) q^{(3)}(0)+W_{20}^{(3)}(0) \bar{q}^{(3)}(0)\right)\right. \\
& +h_{2}\left(W_{11}^{(1)}(0) q^{(3)}(0)+\frac{1}{2} W_{20}^{(1)}(0) \bar{q}^{(3)}(0)+W_{11}^{(3)}(0) q^{(1)}(0)\right. \\
& \left.+\frac{1}{2} W_{20}^{(3)}(0) \bar{q}^{(1)}(0)\right)+h_{3}\left(\bar{q}^{(1)}(0)\left(q^{(3)}(0)\right)^{2}\right. \\
& \left.\left.+2 q^{(1)}(0) q^{(3)}(0) \bar{q}^{(3)}(0)\right)+3 h_{4}\left(q^{(3)}(0)\right)^{2} \bar{q}^{(3)}(0)\right) \\
& +\bar{q}_{3}^{*}\left(k_{1}\left(2 W_{11}^{(3)}(0) q^{(3)}(0)+W_{20}^{(3)}(0) \bar{q}^{(3)}(0)\right)\right. \\
& +k_{2}\left(W_{11}^{(1)}(0) q^{(3)}(0)+\frac{1}{2} W_{20}^{(1)}(0) \bar{q}^{(3)}(0)+W_{11}^{(3)}(0) q^{(1)}(0)\right. \\
& \left.+\frac{1}{2} W_{20}^{(3)}(0) \bar{q}^{(1)}(0)\right)+k_{3}\left(\bar{q}^{(1)}(0)\left(q^{(3)}(0)\right)^{2}\right. \\
& \left.\left.\left.+2 q^{(1)}(0) q^{(3)}(0) \bar{q}^{(3)}(0)\right)+3 k_{4}\left(q^{(3)}(0)\right)^{2} \bar{q}^{(3)}(0)\right)\right), \\
& W_{20}(\theta)=\frac{i g_{20} q(0)}{\tau_{0} \omega_{0}} e^{i \tau_{0} \omega_{0} \theta}+\frac{i \bar{g}_{02} \bar{q}(0)}{3 \tau_{0} \omega_{0}} e^{-i \tau_{0} \omega_{0} \theta}+E_{1} e^{2 i \tau_{0} \omega_{0} \theta}, \\
& W_{11}(\theta)=-\frac{i g_{11} q(0)}{\tau_{0} \omega_{0}} e^{i \tau_{0} \omega_{0} \theta}+\frac{i \bar{g}_{11} \bar{q}(0)}{\tau_{0} \omega_{0}} e^{-i \tau_{0} \omega_{0} \theta}+E_{2}, \\
& E_{1}=\left(\begin{array}{ccc}
2 i \omega_{0}-a_{11} & 0 & -a_{13}-b_{13} e^{-2 i \tau_{0} \omega_{0}} \\
-a_{21} & 2 i \omega_{0}-a_{22} & -a_{23} \\
-a_{31} & -a_{32} & 2 i \omega_{0}-a_{33}-b_{33} e^{-2 i \tau_{0} \omega_{0}}
\end{array}\right)^{-1} \\
& \times\left(\begin{array}{c}
E_{1}^{(1)} \\
E_{1}^{(2)} \\
E_{1}^{(3)}
\end{array}\right) \\
& E_{2}=-\left(\begin{array}{ccc}
a_{11} & a_{12} & a_{13}+b_{13} \\
a_{21} & a_{22} & a_{23} \\
a_{31} & a_{32} & a_{33}+b_{33}
\end{array}\right)^{-1} \times\left(\begin{array}{c}
E_{2}^{(1)} \\
E_{2}^{(2)} \\
E_{2}^{(3)}
\end{array}\right), \\
& E_{1}^{(1)}=g_{1}\left(q^{(3)}(0)\right)^{2}+g_{2} q^{(1)}(0) q^{(3)}(0), \\
& E_{1}^{(2)}=h_{1}\left(q^{(3)}(0)\right)^{2}+h_{2} q^{(1)}(0) q^{(3)}(0), \\
& E_{1}^{(3)}=k_{1}\left(q^{(3)}(0)\right)^{2}+k_{2} q^{(1)}(0) q^{(3)}(0), \\
& E_{2}^{(1)}=2 g_{1} q^{(3)}(0) \bar{q}^{(3)}(0)+g_{2}\left(q^{(1)}(0) \bar{q}^{(3)}(0)+\bar{q}^{(1)}(0) q^{(3)}(0)\right) \text {, } \\
& E_{2}^{(2)}=2 h_{1} q^{(3)}(0) \bar{q}^{(3)}(0)+h_{2}\left(q^{(1)}(0) \bar{q}^{(3)}(0)+\bar{q}^{(1)}(0) q^{(3)}(0)\right), \\
& E_{2}^{(3)}=2 k_{1} q^{(3)}(0) \bar{q}^{(3)}(0)+k_{2}\left(q^{(1)}(0) \bar{q}^{(3)}(0)+\bar{q}^{(1)}(0) q^{(3)}(0)\right) \text {. }
\end{aligned}
$$

\section{Conflicts of Interest}

The author declares that there are no conflicts of interest regarding the publication of this paper.

\section{Acknowledgments}

This work was supported by Natural Science Foundation of the Higher Education Institutions of Anhui Province (KJ2015A144).

\section{References}

[1] T. Zhang, X. Meng, T. Zhang, and Y. Song, "Global dynamics for a new high-dimensional SIR model with distributed delay," Applied Mathematics and Computation, vol. 218, no. 24, pp. 11806-11819, 2012.

[2] Y. Xiao, T. Zhao, and S. Tang, "Dynamics of an infectious diseases with media/psychology induced non-smooth incidence," Mathematical Biosciences and Engineering. MBE, vol. 10, no. 2, pp. 445-461, 2013.

[3] W. Qin, S. Tang, and R. A. Cheke, "Nonlinear pulse vaccination in an SIR epidemic model with resource limitation," Abstract and Applied Analysis, vol. 2013, Article ID 670263, 13 pages, 2013.

[4] T. Zhang, R. Kang, K. Wang, and J. Liu, "Global dynamics of an SEIR epidemic model with discontinuous treatment," Advances in Difference Equations, vol. 2015: Article 361, 16 pages, 2015.

[5] X. Wang, "An SIRS Epidemic Model with Vital Dynamics and a Ratio-Dependent Saturation Incidence Rate," Discrete Dynamics in Nature and Society, vol. 2015, Article ID 720682, 9 pages, 2015.

[6] Y. Tian and X. Liu, "Global dynamics of a virus dynamical model with general incidence rate and cure rate," Nonlinear Analysis. Real World Applications, vol. 16, pp. 17-26, 2014.

[7] D. Zhao and S. Yuan, "Persistence and stability of the diseasefree equilibrium in a stochastic epidemic model with imperfect vaccine," Advances in Difference Equations, vol. 2016: Article ID 280, 14 pages, 2016.

[8] L. Liu, "A delayed SIR model with general nonlinear incidence rate," Advances in Difference Equations, vol. 2015: Article 329, 11 pages, 2015.

[9] Y. N. Xiao and L. S. Chen, "An SIS epidemic model with stage structure and a delay," Acta Mathematicae Applicatae Sinica, vol. 18, pp. 607-618, 2002.

[10] Q. Liu and P. Van Mieghem, "Evaluation of an analytic, approximate formula for the time-varying SIS prevalence in different networks," Physica A. Statistical Mechanics and its Applications, vol. 471, pp. 325-336, 2017.

[11] J. Wang, "Analysis of an SEIS Epidemic Model with a Changing Delitescence," Abstract and Applied Analysis, vol. 2012, Article ID 318150, 10 pages, 2012.

[12] Y. Enatsu, E. Messina, Y. Muroya, Y. Nakata, E. Russo, and A. Vecchio, "Stability analysis of delayed SIR epidemic models with a class of nonlinear incidence rates," Applied Mathematics and Computation, vol. 218, no. 9, pp. 5327-5336, 2012.

[13] F.-F. Zhang, Z. Jin, and G.-Q. Sun, "Bifurcation analysis of a delayed epidemic model," Applied Mathematics and Computation, vol. 216, no. 3, pp. 753-767, 2010.

[14] W. Wang and L. Chen, "Stability and Hopf Bifurcation Analysis of an Epidemic Model by Using the Method of Multiple 
Scales," Mathematical Problems in Engineering, vol. 2016: Article 2034136, 8 pages, 2016.

[15] J.-J. Wang, J.-Z. Zhang, and Z. Jin, "Analysis of an SIR model with bilinear incidence rate," Nonlinear Analysis. Real World Applications, vol. 11, no. 4, pp. 2390-2402, 2010.

[16] J.-Z. Zhang, Z. Jin, Q.-X. Liu, and Z.-Y. Zhang, "Analysis of a delayed SIR model with nonlinear incidence rate," Discrete Dynamics in Nature and Society, vol. 2008, Article ID 636153, 16 pages, 2008.

[17] L. Wen and X. Yang, "Global stability of a delayed SIRS model with temporary immunity," Chaos, Solitons \& Fractals, vol. 38, no. 1, pp. 221-226, 2008.

[18] Y. N. Kyrychko and K. B. Blyuss, "Global properties of a delayed SIR model with temporary immunity and nonlinear incidence rate," Nonlinear Analysis. Real World Applications, vol. 6, no. 3, pp. 495-507, 2005.

[19] P. Yongzhen, S. Li, C. Li, and S. Chen, "The effect of constant and pulse vaccination on an SIR epidemic model with infectious period," Applied Mathematical Modelling. Simulation and Computation for Engineering and Environmental Systems, vol. 35, no. 8, pp. 3866-3878, 2011.

[20] Y. Song, M. Han, and J. Wei, "Stability and Hopf bifurcation analysis on a simplified BAM neural network with delays," Physica D. Nonlinear Phenomena, vol. 200, no. 3-4, pp. 185-204, 2005.

[21] B. D. Hassard, N. D. Kazarinoff, and Y. H. Wan, Theory and Applications of Hopf Bifurcation, Cambridge University Press, Cambridge, UK, 1981.

[22] M. Ferrara, L. Guerrini, and G. M. Bisci, "Center Manifold Reduction and Perturbation Method in a Delayed Model with a Mound-Shaped Cobb-Douglas Production Function," Abstract and Applied Analysis, vol. 2013, Article ID 738460, 6 pages, 2013.

[23] X.-Y. Meng, H.-F. Huo, X.-B. Zhang, and H. Xiang, "Stability and Hopf bifurcation in a three-species system with feedback delays," Nonlinear Dynamics, vol. 64, no. 4, pp. 349-364, 2011.

[24] C. Xu, X. Tang, and M. Liao, "Stability and bifurcation analysis of a six-neuron BAM neural network model with discrete delays," Neurocomputing, vol. 74, no. 5, pp. 689-707, 2011. 


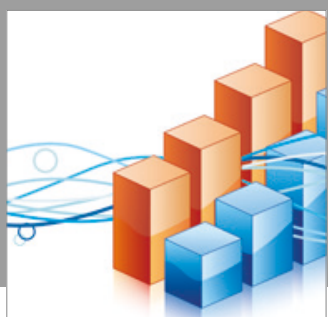

Advances in

Operations Research

vatersals

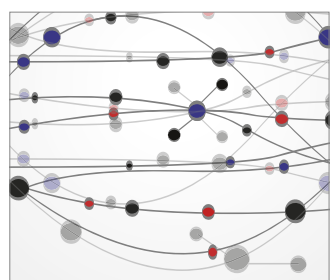

\section{The Scientific} World Journal
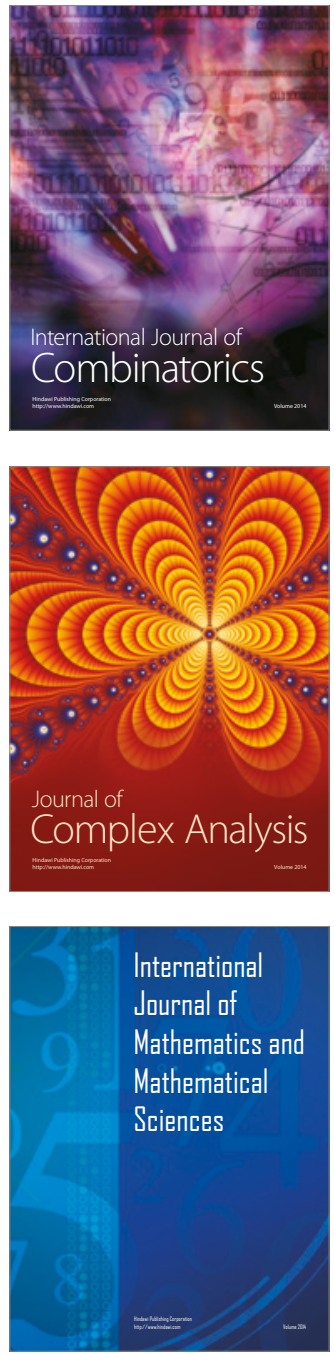
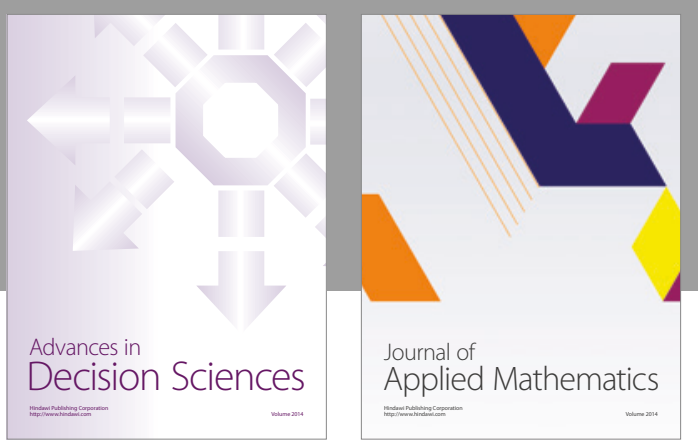

Algebra

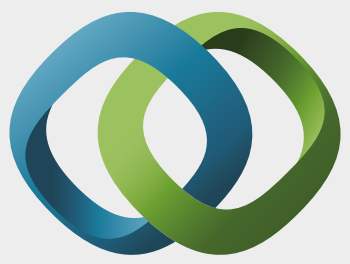

\section{Hindawi}

Submit your manuscripts at

https://www.hindawi.com
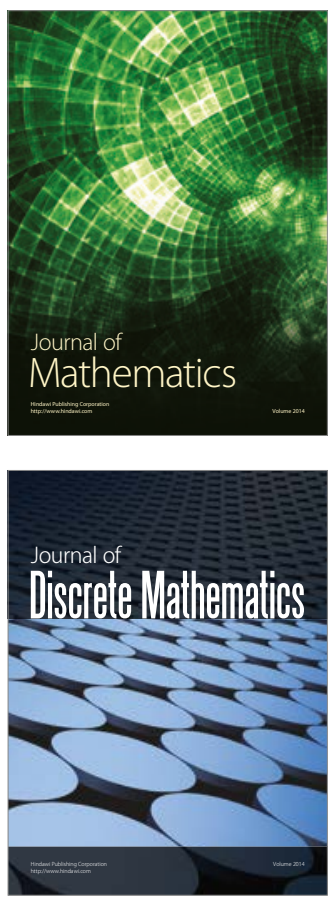

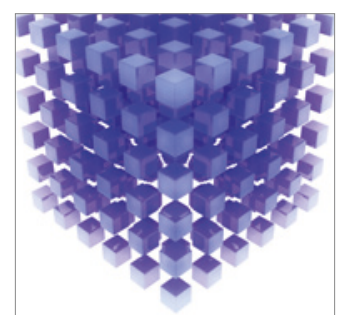

Mathematical Problems in Engineering
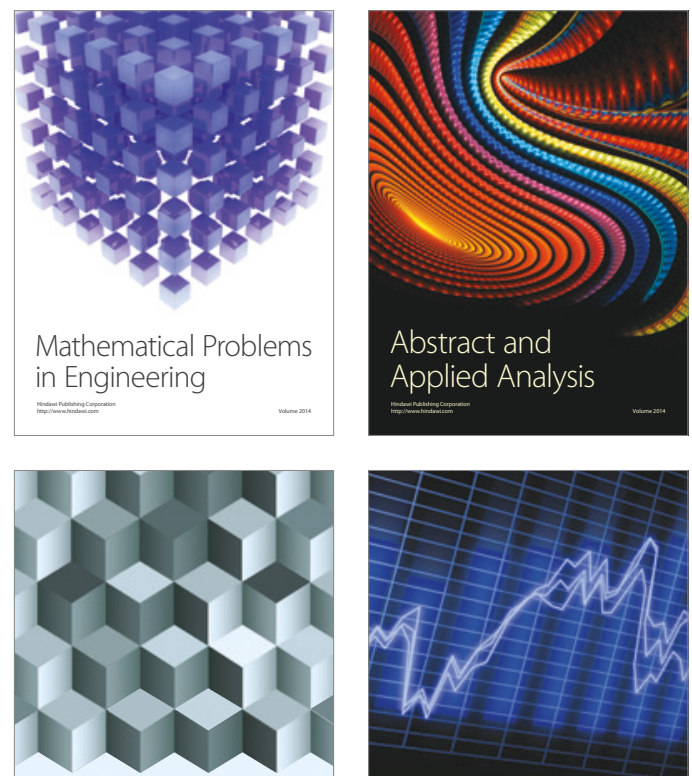

Journal of

Function Spaces

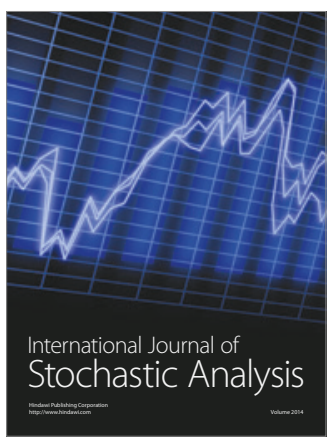

Probability and Statistics
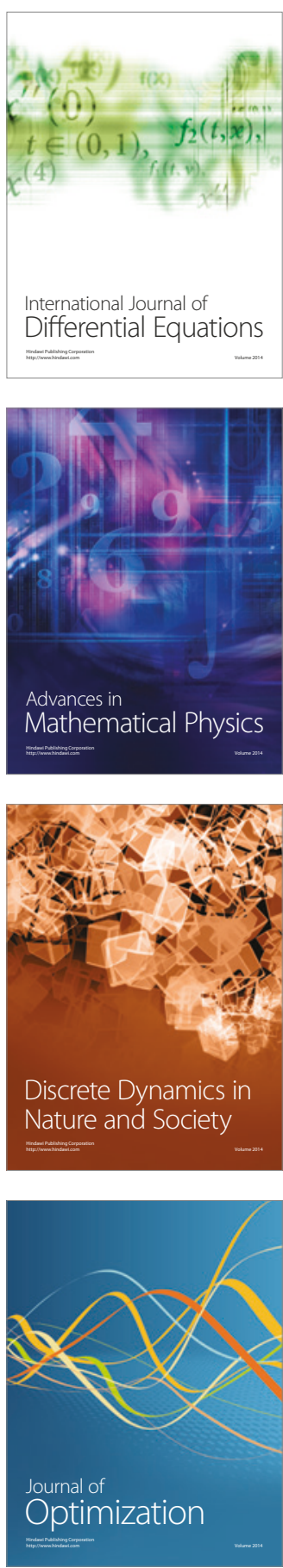